\title{
Physical Activity Levels at Work and Leisure Among Kindergarten Workers
}

\author{
Pål Lagestad, Karin Kippe \\ Department of Sport and Physical Education, North University, Levanger, Norway
}

Email address:

pal.a.lagestad@nord.no (P. Lagestad), Karin.o.kippe@nord.no (K. Kippe)

\section{To cite this article:}

Pål Lagestad, Karin Kippe. Physical Activity Levels at Work and Leisure Among Kindergarten Workers. Science Journal of Public Health. Vol. 4, No. 3, 2016, pp. 147-154. doi: 10.11648/j.sjph.20160403.12

Received: February 1, 2016; Accepted: April 1, 2016; Published: April 25, 2016

\begin{abstract}
Background: The low percentage of adults fulfilling the health recommendations standard of physical activity in Norway and in countries all over the world, is a major concern. The study examine how physical activity during work hours contributes to physical activity among kindergarten workers, and how these workers estimate their physical activity level. Methods: Accelerometers were used for seven days to derive percentages of time spent in moderate-to-vigorous physical activity (MVPA) among forty-six randomly selected kindergarten workers in Norway. A questionnaire was used to measure the subjects' estimation of their own activity. Results: The study found that kindergarten workers achieved 57\% of their MVPA during work on work days and 44\% of their weekly MVPA through their work. Furthermore, the subjects underestimated their own MVPA during work, but not during leisure. Conclusion and recommendations: The results suggest that physically active work may be a major arena for daily physical activity, and that workers in physically active professions may be unaware of the positive outcomes regarding the contribution of work time to the daily MVPA level. The results point to the importance of making people aware of the positive outcome of physically demanding professions in relaton to fulfill the physical activity reccomendation.
\end{abstract}

Keywords: Moderate-to-Vigorous Physical Activity, Work Time, Health Recommendation, Estimation

\section{Introduction}

Research has highlighted the importance of fulfilling physical activity recommendations so as to achieve good health. A 2014 study showed that nearly two of three Norwegian adults did not fulfill the Norwegian health recommendations standard of 150 minutes of moderate-tovigorous physical activity (MVPA) per week [1]. The low percentage of adults fulfilling this standard of 150 MVPA in Norway and in countries all over the world is a major concern. In general, adults spend approximately half of their time awake at work. Determining how the time spent at work among various professions contributes to the fulfillment of physical activity health recommendation standards is thus important.

A recent study showed that employees at a kindergarten were more physically active than their peers [2], with four out of five of the employees at the kindergarten fulfilling the health recommendations for physical activity. Only one out of three of Norwegian women of the same age in the general population fulfilling these recommendations [1]. Physically demanding work may have contributed to the high level of fulfillment of health recommendations among the kindergarten employees, but how much of this physical activity was obtained during work time, is unknown. A literature search reveals a lack of research on the importance of the contribution of work to the fulfillment of the health recommendations for physical activity.

\subsection{Litterature Review}

In their review study, which included articles published up to 2009 on the measurement of physical activity levels at work, Castillo-Retamal and Hinckson [3] found that the most common method of data collection was through self-report surveys or questionnaires, as well as with pedometers. The authors concluded that these studies showed that physical activity levels at work were low, and that sedentary behavior was high. A study by McCrady and Levine [4] supports this 
conclusion. Nevertheless, such measurements are somewhat insufficient in determining the contribution of physical activity carried out at work. Research has showed that self-report instruments for measuring physical activity may overestimate the proportion who fulfill the health recommendations $[5,6]$. Using an accelerometer is a more valid and common method of measuring the fulfillment of physical activity recommendations among populations. The procedures are standardized, and the measures are objective, unlike selfreported physical activity, which seems to pose some challenges regarding validity and reliability [7]. However, few studies have been conducted using accelerometers to investigate the level of physical activity at work. Studies using accelerometers have mostly included validation studies and explored activity levels among groups with different types of diseases, diagnoses, ages, and levels of obesity.

Some new studies about the level of physical activity at work have been published after Castillo-Retamal and Hinckson [3] conducted their review study. Most of these studies used accelerometers, but the results were rather ambivalent. These research studies indicate that work time may contribute to obtaining the minimum level of physical activity during the day, but the level of physical activity achieved at work seems to vary among professions and with the sedentary characteristics of the profession. Office work and other types of sedentary work seem to contribute little to the general physical activity level of individuals. When Thorp et al. [8] used accelerometers among customer service workers and call center workers, they found that call center workers were generally the most sedentary and least physically active at work, whereas customer service workers were typically the least sedentary and the most active at work. Furthermore, Thorp et al. [8] found that the call center workers and customer service workers spent $1.9 \%$ of their time in MVPA during work hours and $4.3 \%$ of their time in MVPA during nonwork hours. Clemes, O'Connell \& Edwardson [9] studied sedentary behavior and physical activity during and outside work hours among full-time office workers. They found that the office workers accumulated significantly higher levels of sedentary behavior on work days in comparison to nonwork days. Parry and Straker [10] found that sedentary time accounted for $81.8 \%$ of work hours, which was significantly greater than sedentary time during nonwork time. During work hours, the MVPA level accounted for only $2.9 \%$ of the time. The authors concluded that office work is characterized by sustained sedentary time and contributes substantially to the overall sedentary exposure of office workers.

The studies discussed in the preceding paragraph all focused on sedentary work. Another example is a study by McCrady and Levine [4], who examined whether people with sedentary jobs were equally inactive during their work days and leisure days. Not surprisingly, they found that work days were associated with more sitting and less walking and standing time than leisure days. Instead of studying how little sedentary professions contribute to daily physical activity levels, scholars may want to shift their focus to studying the importance of physical activity in more physically demanding professions. Such research is lacking as of yet. Using pedometers, Miller and Brown [11] found that workers in managerial and professional occupations reported more time sitting at work and lower weekday step counts than technical and blue-collar workers. Their study indicates that physically demanding professions may contribute more to daily physical activity than sedentary jobs. Other studies in which accelerometers were not used also provide support for this argument. Cheung and Chow [12] found that physical education (PE) teachers had higher step counts during work hours compared with non-PE teachers. Sena, Pontes, Ferreira and Silva [13] found that in relation to the pattern of physical activity analyzed by the International Physical Activity Questionnaire (IPAQ), all of the postmen in their study were classified as active, whereas only $19 \%$ of the taxi drivers were classified as such. Tigbe, Lean and Granat [14], using activPAL, studied the physical activity levels of active (walking) postal delivery workers and inactive administrative postal workers. They found that delivery staff spent substantially longer time upright and walking and amassed more steps on work days than office staff. Nevertheless, one may question whether pedometers and self-reported activity are appropriate tools for measuring activity levels from a health perspective.

A study using accelerometers among auxiliary nurses and secretaries found that physical activity levels were higher among auxiliary nurses than secretaries [15], but the study included only eight individuals in each group. Wong, Gilson, Bush and Brown [16] found that among male transport workers, sedentary time was significantly lower when employees were working, than when not working on work days. No significant differences were found for time spent in moderate physical activity. Even though transport workers engage in some types of physical activity, such as lifting and carrying, in their work, one may question how physically demanding their work is in general.

Some studies have compared workers from physically demanding occupations with workers from less physically demanding occupations, and their findings support the argument that physically demanding occupations may contribute substantially to the fulfillment of the health recommendations for physical activity. For example, a bachelor's thesis showed that a group of carpenters and a group of academics had the same activity levels during the day but that the carpenters' activity levels were significantly higher during work time [16]. Furthermore, substantially more carpenters fulfilled the health recommendations for physical activity.

Although the findings of previous studies indicate that physical activity levels at work vary among professions and that physically demanding occupations may contribute substantially to the fulfillment of the health recommendations for physical activity, these findings do not provide knowledge about the exact importance work time has in relation to the fulfillment of the health recommendations for physical activity. A recent study by Arias, Caban-Martinez, 
Umukoro, Okechukwu \& Dennerlein [17] reported that physical activity in work contributed to $65 \%$ of the time (minutes) spent in moderate physical activity and to $29 \%$ of the time spent in vigorous physical activity, whereas physical activity outside work contributed to $35 \%$ of the total time (minutes) spent in moderate physical activity and $71 \%$ of the total time spent in vigorous physical activity. However, the study did not report the daily MVPA in relation to the health recommendations for physical activity, and the data make it difficult to state the exact contribution of daily MVPA.

\subsection{Research Question}

Thorp et al. [8] put forward the argument that the workplace is a key setting for prolonged sedentary time, especially for some occupational groups. Previous research has also suggested that the workplace may be a key setting in relation to the fulfillment of the health recommendations for MVPA. Working in a kindergarten can be a physically demanding profession that involves a great deal of standing up, sitting down, and walking, which may explain the high MVPA levels found among kindergarten employees [2]. The aim of the present study is to examine how physical activity during work hours among kindergarten workers contributes to daily and weekly physical activity levels in relation to MVPA and CPM. Furthermore, this study examines how these workers estimate their own MVPA levels during work hours and nonwork hours.

\section{Methods}

\subsection{Design}

The ActiGraph GT1M accelerometer was used in measuring physical activity levels among kindergarten workers in a cross-sectional field study. The validity and reliability of this method in measuring physical activity in relation to the fulfillment of health recommendations among populations have been previously tested [1]. Employing this method makes it possible to compare the subjects' activity levels (MVPA and CPM) with the recommended levels and with other populations. Furthermore, the subjects answered a questionnaire that included the following question: How many minutes do you estimate you spent in moderate-tovigorous physical activity on the following day? The subjects estimated the MVPA on each of the seven days they were wearing accelerometer.

\subsection{Subjects}

Only kindergarten workers with full-time employment were selected from the population. Seven kindergartens among the 32 kindergartens in Levanger (municipality), Norway, were randomly selected among the different types of kindergartens (farm or outdoor kindergartens, music kindergartens, ordinary kindergartens). Forty-six employees were randomly selected to wear an accelerometer for a whole week, from Monday to Sunday evening. This was an ordinary work week, working 7.5 hours in general. Everyone agreed to participate in the study. Valid data from both the accelerometer study and the questionnaire were not obtained for 3 subjects. Of these 43 subjects, there were 2 males and 41 females, aged $41.6 \pm 10.2$ years. The selected kindergarten workers had completed various levels of education, with 17 educated as preschool teachers (university college degree), 12 educated as child and youth workers (high school level), and 14 with no special education in childcare. 12 of the subjects working on farm or outdoor kindergartens, 8 at music kindergartens, and 23 at ordinary kindergartens. The subjects were fully informed about the protocol before participating in this study, and informed consent was obtained prior to all testing from all subjects in accordance with the recommendations of the local ethics committee and current ethical standards in sports and exercise research. Approval to use the data and conduct the study was given by the Norwegian Social Science Data Services (NSD).

\subsection{Procedures}

The subjects each wore an accelerometer for a whole week, from Monday to Sunday evening, so as to measure their weekly physical activity levels. They were informed regarding how to wear the accelerometer on the waist on the right side, and instructed to wear the accelerometer all day except while sleeping, taking a shower, or swimming. The accelerometer recorded data in 1-minute intervals, providing the number of counts for each minute for the 7-day period. At the end of the 7 days, we retrieved the accelerometer from the subjects and downloaded the data onto the ActiLife software program. For data to be considered valid, 600 minutes each day was required, and periods over 60 minutes with no activity were removed in accordance with the test protocol [1]. The subjects had to have at least 4 valid days to be included in the study. Only work days were included in the analysis, with the CPM and MVPA values during work being filtered so as to make it possible to find the exact CPM and MVPA during work time and leisure on work days and on the weekend. To do so, the data set was parsed into two sets of data, one with the MVPA and CPM during work time, and the other with the MVPA and CPM during leisure time. In accordance with Norwegian population studies, the MVPA level was defined as more than 2019 CPM [1]. This is the cut point set called Troiano [19] in ActiLife. The questionnaire was pretested before the subjects answered the questions. Of the 46 kindergarten workers who were randomly selected to participate in this study, valid data from both the accelerometer study and the questionnaire were not obtained from 3 of them because 1 subject forgot to put on the accelerometer, 1 subject forgot to fill out the questionnaire, and 1 subject became ill during the week of testing. Hence, valid data from both the accelerometer study and the questionnaire were obtained for 43 subjects.

\subsection{Statistical Analyses}

All results are presented as mean $\pm \mathrm{SD}$ values. A paired sample $t$ test was performed to compare the differences 
between CPM and MVPA during work time and leisure time and between the subjects' estimation of MVPA and the real MVPA. The level for significance was set at $p<.05$. Statistical analysis was performed with Statistical Product and Service Solutions (SPSS) software, version 21.0 (SPSS, Inc., Chicago, IL).

\section{Results}

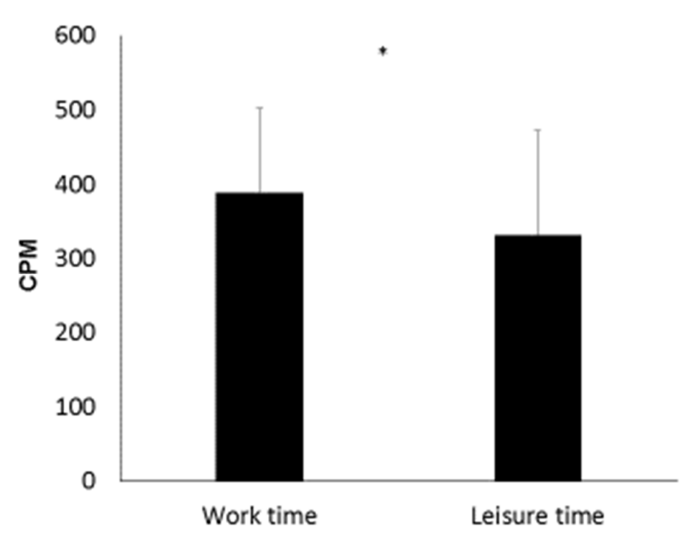

Figure 1. Daily counts per minute (CPM) during work time and leisure on work days. *Significant differences between work time and leisure at the 05 level.

Figure 1 shows a higher CPM of 55 counts during work time than during nonwork time on work days. The CPM is $14.5 \%$ higher during work time compared with the CPM during leisure time. The difference between the CPM during work time and the CPM during leisure time is statistically significant $(t=3.6, p=.006)$. In fact, the employees achieved $54 \%$ of their daily CPM through their work on work days. The CPM value allows comparisons to be made with the CPM values reported in other studies. However, the most common and valid measure of physical activity in relation to the fulfillment of physical activity recommendations is the MVPA. The MVPA values in work and leisure are presented in Figure 2.

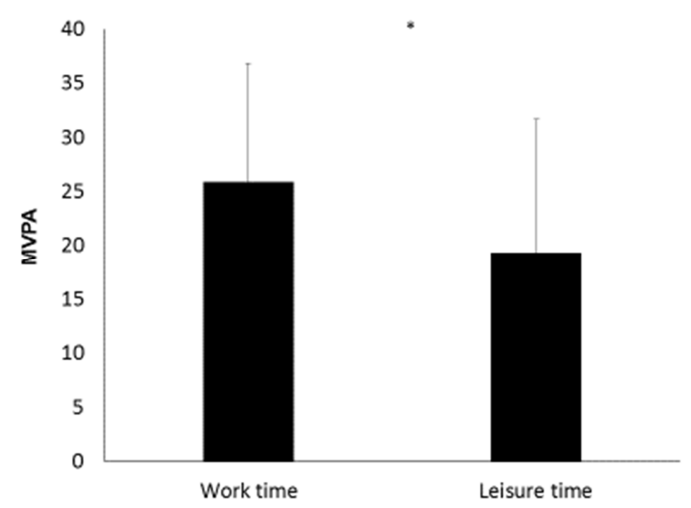

Figure 2. Daily moderate-to-vigorous physical activity (MVPA; >2019) during work time and leisure on work days. *Significant differences between work time and leisure at the 05 level.

Figure 2 shows a higher MVPA of 6.5 minutes during work time than during leisure time on work days. The MVPA is $25.1 \%$ higher during work time compared with the MVPA during leisure time. The difference between MVPA during work and MVPA during leisure on work days is statistically significant $(t=3.9, p=.000)$. In other words, the kindergarten employees achieved $57 \%$ of their daily MVPA on work days through their work. Figure 3 shows the subjects' estimation of their MVPA during work time and leisure time on work days, compared with the objective measures of MVPA during these two periods.

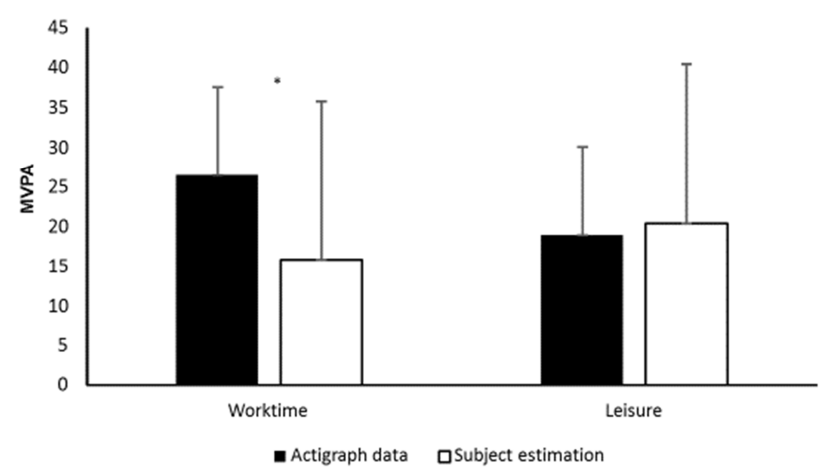

Figure 3. Objective (accelerometer-based) daily moderate-to-vigorous physical activity (MVPA; >2019) data and subject-estimated MVPA data during work time and leisure on work days. *Significant differences between objective accelerometer data and subjects' estimation at the .05 level.

Figure 3 shows a significant difference between the real MVPA during work time and the subjects' estimation of their own MVPA $(t=2.3, p=.028)$. In addition, this figure shows that the subjects underestimated their MVPA during work. The subjects also tended to overestimate their MVPA during leisure, but the difference is very small and not significant. The high standard deviation associated with the subjects' estimation in Figure 3 shows that the subjects' estimation of their own activity level varies greatly.

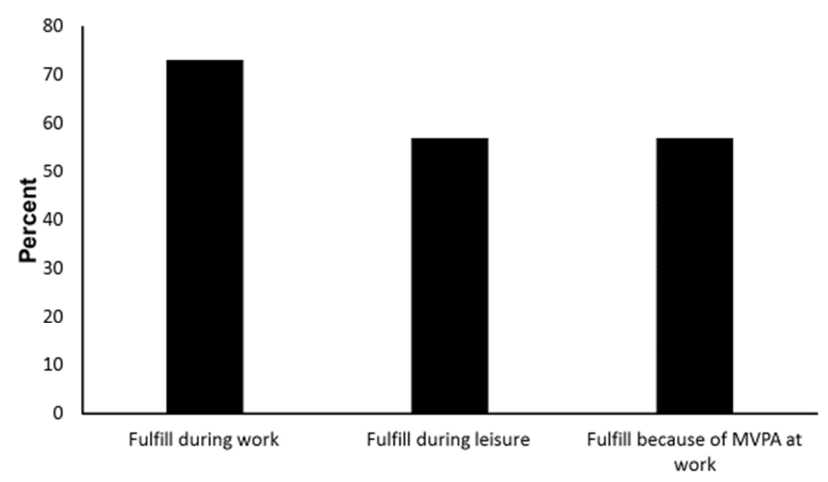

Figure 4. Percentage of workers that fulfill the weekly 150 minutes of moderate and/or vigorous physical activity during work time, leisure time, and because of the contribution of physical activity at work.

To calculate the relative contributions of work to the fulfillment of physical activity health recommendation standards, it would be helpful to only look at kindergarten workers who have reached the guidelines ( 86 percent), and then calculate the relative contributions of work according to the to the fulfillment of physical activity health recommendation standard. The results are presented in figure 4 . 
Figure 4 shows that $73 \%$ of the kindergarten workers who fulfilled the weekly MVPA recommendation, fulfilled it just with being physical active during work. $57 \%$ fulfilled the MVPA recommendation during leisure time. However, it is appropriate to emphasize that everyone that fulfilled the MVPA recommendation during leisure time, also fulfilled the recommendation during work. Furthermore, $57 \%$ fulfilled the MVPA recommendation because they were physical active during work.

Figures 1 through 3 only show the activity levels of the subjects only on work days. To determine the contribution of the MVPA during work in relation to the MVPA during the whole week, knowledge of the activity levels of the subjects on the weekend is recommended. The data are presented in Table 1.

Table 1. CPM (counts per minute) and MVPA (moderate-to-vigorous physical activity) during the weekend.

\begin{tabular}{lll}
\hline & Mean (SD) & No. of subjects \\
\hline CPM weekend & $364(172)$ & 43 \\
MVPA weekend & $45(28)$ & 43 \\
\hline
\end{tabular}

Table 1 shows that the CPM during the weekend is 364 , whereas the MVPA during the weekend is 45 . The standard deviation values show that both the CPM and the MVPA vary among the kindergarten workers in the weekend.

\section{Discussion}

\subsection{The Importance of Physical Activity During Work}

Figure 1 shows that the CPM is $14.5 \%$ higher during work time compared with the CPM during leisure time. Furthermore, Figure 2 shows that the MVPA is $25.1 \%$ higher during work time compared with the MVPA during leisure time. In other words, the kindergarten employees in the present study, achieved $54 \%$ of their CPM and $57 \%$ of their MVPA through their work on work days. If it is assumed that the subjects generally slept for 8 hours per day, this indicates that more high intensive activity (moderate and vigorous activity) occurred during work time, than during leisure time. Furthermore, figure 4 showed that $57 \%$ only fulfilled the MVPA recommendation because they were physical active during work. The results in the present study suggest that workers in physically demanding professions achieve much of their activity level during work. The findings are supported by those of previous studies highlighting the importance of physical activity during work [17, 18].

In their review study of articles, published up to 2009 , focusing on the measurement of physical activity levels, Castillo-Retamal and Hinckson [3] concluded that these articles showed that physical activity levels at work were low, and that sedentary behavior levels were high. However, a great deal of these articles examined sedentary professions and were also based on self-report questionnaires and pedometer readings. It seems as though researchers have focused on studying how little sedentary work contributes to the daily MVPA levels among call center workers and customer service workers [8], office workers [9, 10], and other workers who are chairbound most of the work time [4]. Not surprisingly, these studies found that sedentary work contributed little to the daily physical activity level. Such studies may be useful in some respects. However, when these studies report that $71 \%$ [9] to $81.8 \%$ [10] of the work time was categorized as sedentary, this finding has minor value if the purpose is to examine the contribution of physical activity during work. When Thorp et al. [8] and Parry and Straker [13] showed that workers in sedentary occupations spent $1.9 \%$ and $2.9 \%$, respectively, of the work time at the MVPA level, their results contribute little to study the potential work has to the contribution of MVPA. However, some other studies using self-report questionnaires and pedometer readings have studied the contribution of physical activity at work in greater detail by comparing sedentary professions with more physically demanding professions [11, $12,13,14]$. All these studies showed that physically demanding professions contributed more to daily physical activity than less physically demanding jobs. However, accelerometers were not used in these studies; thus, it is difficult to conclude how physically demanding professions affected the MVPA during work time. The few studies using accelerometers to study the contribution of work time to the MVPA level indicate that work time in physically demanding professions may contribute substantially, but these studies do not indicate how much physical demanding professions contributes to the daily MVPA level.

A starting point for the present study was a previous study on the same group of kindergarten workers, showing that employees at kindergartens were more physically active than their peers in the general population [2]. The results of this study suggested that physical activity during work contributed substantially to the high physical activity level found among the kindergarten workers. The results of Figures 1 and 2 of the present study support this statement and provide knowledge about the exact contribution of CPM and MVPA during work. The unpublished data [2] showed that the kindergarten workers' CPM was 370 (SD 120), which was higher than their peers' (general population) CPM of 330 (SD 135) according to a national population study [1]. However, the present study shows that the kindergarten workers' CPM on work days was 388 (SD 135) during work time and 332 during leisure time. It is striking that the kindergarten workers' CPM during leisure in the present study, is nearly the same as their peers' general CPM [1]. The findings displayed in Figures 1 and 2 in the present study also show that working at a kindergarten facilitates physical activity during work, and that it is the activity during work that makes the difference.

Figure 4 in the present study shows that $73 \%$ of the kindergarten workers who fulfilled the weekly MVPA recommendation, fulfilled it just with being physical active during work, while $57 \%$ only fulfilled the MVPA recommendation because they were physical active during work. Based on our understanding, achieving MVPA during work is essential in fulfilling the recommendations for daily 
MVPA. This finding is supported by Arias et al. [18], who found that $73 \%$ of the construction workers met the recommended weekly physical activity level through their activity during work, and that $31 \%$ of the construction workers met the daily MVPA recommendation through physical activity during leisure.

Table 1 in the present study shows that the daily CPM and MVPA values during weekends were 365 and 45, respectively. The findings in Figure 2 and Table 1 show that each work day contributes just as much as days on the weekends. During the 5 work days, work contributes with 130 minutes, or $44 \%$ of the total weekly MVPA (Figure 2, Table 1), which is less than the $65 \%$ of the total weekly MVPA reported for the construction workers in the study by Arias et al. [18]. It may be argued that construction workers have more physically demanding work than kindergarten workers, which may explain the finding. In the present study, kindergarten workers achieved 130 MVPA during work time, which is $87 \%$ of the international recommendation of 150 MVPA during a week.

The results of the present study indicate that physically demanding occupations contribute substantially to the MVPA level.

\subsection{Underestimation of One's Own Physical Activity Level}

Figure 3 in the present study shows that there is a significant difference between the real MVPA level during work time and the subjects' estimation of their own MVPA level, with the subjects underestimating their MVPA during work but not during leisure. This finding of underestimation instead of overestimation is in contradiction to other studies $[5,18,20,21,22,23]$. Arias et al. [18] studied the difference between workers' self-reported estimation of the MVPA and accelerometer-measured MVPA. Their findings showed that the workers reported their MVPA to be approximately six times higher during work, and five times higher outside work compared with the accelerometer-measured MVPA. Boon, Hamlin, Steel \& Ross [24] also found that self-report questionnaires tended to overestimate activity levels, but "only" by approximately $165 \%$.

The large differences between the huge overestimation reported in the study by Arias et al. [18] and the present study may be attributed to different questions regarding the estimation. In the study by Arias et al. [18], the participants were asked how much time they spent doing moderate and vigorous activities (two items) for at least 10 minutes at a time while at work in the past 7 days and then while not at work in the past 7 days. The responses were summed to reflect the total time (minutes) spent in moderate activity and the total time (minutes) spent in vigorous physical activity during work and leisure. In the present study, the workers were asked to estimate how many minutes they were in moderate or vigorous activity on each day of the 7 days they wore accelerometers. These two questions represent two completely different ways of estimating one's own MVPA level. Our results indicate that the strategy used in the present study may be more reliable and valid than other studies, because the estimations were more realistic than the estimations reported in other studies. Nevertheless, the large standard deviations in the estimation rates in Figure 3 in the present study, also show a huge variation in the estimation of the MVPA level - a variation that is much larger than the objectively measured MVPA. This finding is supported by Arias et al. [18], who found that the standard deviations were even higher than the mean MVPA. In Figure 3 in the present study, the mean estimation of MVPA during leisure is approximately 20, but the estimation varies from 0 to 40 MVPA. This finding indicates that estimating one's own MVPA level is difficult, and that many fail to estimate their own activity level accurately. The results in Figure 3 indicate that this is especially problematic in relation to work.

Several reasons may explain why the main finding of the present study (underestimation of physical activity levels) contradicts those of other studies. As discussed earlier, the questions that form the basis of the estimations seem to be completely different in the various studies. Another possible explanation is the fact that most of the subjects reached the weekly MVPA recommendation. In studies with reported overestimation, most subjects had not reached the MVPA recommendation. In the present study, $86 \%$ of the subjects met the MVPA recommendation. It may be argued that it is easier to overestimate low levels of physical activity than high levels of physical activity. A study has also found that overestimation may be gender related. Watkinson et al. [23] found that males were more likely to overestimate their physical activity levels. In the present study, approximately $95 \%$ of the subjects were women. Arvidsson et al. [5] also found that such overestimation varied among different groups. This finding may help explain the absence of overestimation in Figure 3. Finally, estimation of one's own activity level is challenging. During the data collection in the present study, many subjects reported that estimation of one's own MVPA level was difficult. This difficulty may cause huge variations in the estimations. How the estimation is done and when the estimation takes place, may also affect the estimation.

The results in the present study indicate that workers in physically active professions may be unaware of the positive outcomes regarding the contribution of work time to the daily MVPA level. Furthermore, the results point to the importance of making people aware of the positive outcome of physically demanding professions in relaton to fulfill the physical activity reccomendation. The findings may contribute in increasing the status of such professions in the future.

\section{Limitation}

The present study included approximately 95 percent woman, and only 5 percent men. However, such a design complies with gender patterns in kindergarten in Norway, where over 90 percent employees are women. It is appropriate to point out that research show that high occupational physical activity is not necessarily protective of health. Richard, Martin, 
Wanner, Eichholzer and Rohrmann [25] showed that occupational PA actually seemed to increase risk of early death among men. However, in women there was no association between physical activity at work and mortality. The researchers suggest that high occupational physical activity probably characterizes different physical demands than high occupational physical activity in women. Holterman [26] point to increased risk for cardiovascular disease among male blue collar workers with high occupational physical activity. High occupational physical activity may be related to health risks, but only among male workers. The present study included mainly women. High physical activity during work in kindergartens may not have such negative health effects as Richard et al. [25] and Holterman [26] suggests. In contradiction, as Thorp et al. [8] suggest and the results indicate - the workplace may be a key setting for kindergarten workers in relation to the achievement of physical activity in an inactive life. As discussed earlier, the measure of the subjects estimation of their physical activity level were not based on the same questions as other questions. Such a strategy make it difficult to compare the present study with other studies, and may create a validity problem. However, it has been argued that the strategy used in the present study gave better estimation of the subjects activity levels, than questions used in other studies.

\section{Conclusion}

This study presents new knowledge about how physical activity during work hours may contribute significantly to daily physical activity levels, and to the fulfillment of daily physical activity recommendations. The findings show that kindergarten workers achieved $57 \%$ of their MVPA during work on work days, and $44 \%$ of their weekly MVPA through their work. Furthermore, physical activity during work contributes to $87 \%$ of the recommended 150 MVPA per week. The results suggest that physically active work is a major arena for daily physical activity. Thus, it is reasonable to suggest that other physically active professionals (e.g., carpenters and plumbers) will benefit substantially from their work in relation to daily physical activity health reccomendations, even if other research indicate that high occupational physical activity may affect the health among male collar workers negatively. By contrast, previous research has identified sedentary work (e.g., office work) as contributing little to workers' general physical activity levels. Furthermore, the results show that unlike other studies, the subjects in the present study underestimated their MVPA during work. Several reasons may explain this difference. One reason may be that the number of subjects fulfilling the physical activity recommendations was much higher in the present study than in previous studies. It is argued that it is easier to overestimate low levels of physical activity than high levels of physical activity. Another reason may be that males are more likely to overestimate their physical activity levels, and in the present study, approximately $95 \%$ of the subjects were women.
Instead of studying how little physically inactive professions contribute to physical activity levels, the findings highlight the need for studies concerning physical activity levels in other physically demanding professions. Further research is needed to establish a stronger link between activity at work and the fulfillment of daily physical activity in other professions.

\section{References}

[1] Hansen, B. H., Kolle, E., \& Anderssen, S. A. (2014). Fysisk aktivitet blant voksne og eldre i Norge. Opppdaterte analyser basert på nasjonale anbefalinger i 2014 [Physical activity level among adults and older people in Noway. Updated analyses based on national recommendations]. Oslo: Helsedirektoratet.

[2] Kippe, K., \& Lagestad, P. (unpublished). Fysisk aktivitetsnivå blant barnehageansatte [physical activity level among kindergarten empoyees].

[3] Castillo-Retamal, M., \& Hinckson, E. A. (2011). Measuring physical activity and sedentary behaviour At work: a review. Work, 40, 345-57.

[4] McCrady, S. K., \& Levine, J. A. (2009). Sedentariness at work; how much do we really sit? Obesity, 17, 2103-2105.

[5] Arvidsson, D., Leijon, M., Sundquist, J., Sundquist, K., Lindblad, U., \& Bennet, L. (2014). Cross- cultural validation of a simple self-report instrument of physical activity in immigrants from the Middle East and native Swedes. Scandinavian Journal of Public Health, 42, 255-262.

[6] Celis-Morales, C. A., Perez-Bravo, F., Ibañez, L., Salas, C., Bailey, M. E., \& Gill, J. M. (2012). Objective vs. self-reported physical activity and sedentary time: effects of measurement method on relationships with risk biomarkers. PLoS One, 7, 1-10.

[7] Ekelund, U., Tomkinson, G., \& Armstrong, N. (2011). What proportion of youth are physically active? Measurement issues, levels and recent time trends. British Journal of Sports Medicine, 45, 859-65.

[8] Thorp, A. A., Healy, G. N., Winkler, E., Clark, B. K., Gardiner, P. A., Owen, N., \& Dunstan, D. W. (2012). Prolonged sedentary time and physical activity in workplace and non-work contexts: a cross-sectional study of office, customer service and call centre employees. International Journal of Behavavioral Nutrition and Physical Activity, 9, 128-136.

[9] Clemes, S. A., O'Connell, S. E., \& Edwardson, C. L. (2014). Office workers' objectively measured sedentary behavior and physical activity during and outside working hours. Journal of Occupational and Environmental Medicine, 56, 298-303.

[10] Parry, S., \& Straker, L. (2013). The contribution of office work to sedentary behaviour associated risk. BMC Public Health, 13, 1-10.

[11] Miller, R., \& Brown, W. (2004). Steps and sitting in a working population. International Journal of Behavioral Medicine, 11, 219-24.

[12] Cheung, P. Y. P., \& Chow, B. C. (2012). Association of school teachers' occupational and daily Physical activity level in Hong Kong. International Journal of Sport \& Health Science, 10, 23-29. 
[13] Sena, J. E. A, Pontes, L. M., Ferreira, U. M. G., \& Silva, J. M. (2008). Composición corporal y su relación con el nivel de actividad física de axistas y carteros de João Pessoa - PB [Body composition and its relationship with the level of physical activity of taxi drivers and postmen of João Pessoa PB]. Fitness and Performance Journal, 7, 20-25.

[14] Tigbe, W. W., Lean, M. E., \& Granat, M. H. (2011). A physically active occupation does not result in compensatory inactivity during out-of-work hours. Preventive Medicine, 53, $48-52$.

[15] Bravo, M. F., Paredes, F. Z., Rodriguez-Rodriguez, F. J., \& Cristi-Montero, C. (2013). Occupational physical activity and body composition in adult women; pilot study. Nutricion hospitalaria, 28, 1060-1064.

[16] Wong, J. Y. L., Gilson, N. D., Bush, R. A., \& Brown, W. J. (2014). Patterns and perceptions of physical activity and sedentary time in male transport drivers working in regional Australia. Australian and New Zealand Journal of Public Health, 38, 314-320.

[17] Hoel, K. A., \& Øren, T. S. (2013). Fysisk aktivitet hjå akademikarar og tømrarar $i$ Sogndal målt med akselerometer [Physical activity levels among academics and carpenters measured with accelerometers]. Bachelor's thesis. http://www.nb.no/idtjeneste/URN:NBN:no-

bibsys_brage_49993 http://hdl.handle.net/11250/149891. Accessed 11 Nov 2014.

[18] Arias, O. E., Caban-Martinez, A. J., Umukoro, P. E., Okechukwu, C. A., \& Dennerlein, J. T. (2015). Physical activity levels at work and outside of work among commercial construction workers. Journal of Occupational and Environmental Medicine, 57, 73-78.

[19] Troiano, R. P., Berrigan, D., Dodd, K. W., Masse, L. C., Tilert, T., \& McDowell, M. (2008). Physical activity in the United States measured by accelerometer. Med Sci Sports Exerc., 40(1): 181- 188.
[20] Corder, K. V., Sluijs, E. M. V., McMinn, A. M., Ekelund, U., Cassidy, A., \& Griffin, S. J. (2010). Perception versus reality awareness of physical activity levels of British children. American Journal of Preventive Medicine, 38, 1-8.

[21] Sebastião, E., Gobbi, S., Chodzko-Zajko, W., Schwingel, A., Papini, C. B., \& Nakamura, P. M. (2012). The International Physical Activity Questionnaire-long form overestimates selfreported physical activity of Brazilian adults. Public Health, 26, 967-975.

[22] Umukoro, P. E., Arias, O. E., Stoffel, S. D., Hopcia, K., Sorensen, G., \& Dennerlein, J. T. (2013). Physical activity at work contributes little to patient care workers' weekly totals. Journal of Occupational and Environmental Medicine, 55, 63-68.

[23] Watkinson, C., Sluijs, E. M. V., Sutton, S,. Hardeman, W., Corder, K., \& Griffin, S. J. (2010) Overestimation of physical activity level is associated with lower BMI: a cross-sectional analysis. International Journal of Behavavioral Nutrition and Physical Activity, 7, 68-76.

[24] Boon, R. M., Hamlin, M. J., Stee, 1 G. D., \& Ross, J. J. (2010). Validation of the New Zealand Physical Activity Questionnaire (NZPAQ-LF) and the International Physical Activity Questionnaire (IPAQ-LF) with accelerometry. British Journal of Sports Medicine, 44, 741-746.

[25] Richard, A., Martin, B., Wanner, M., Eichholzer, M., \& Rohrmann, S. (2015). Effects of leisure-time And occupational physical activity on total mortality risk in NHANES III according to sex, ethnicity, central obesity, and age. Journal of Physical Activity and Health, 12(2), 184-92.

[26] Holtermann, A. (2015). Occupational and leisure-time physical activity and coronary heart disease. Occupational and Environmental Medicine, 72, 615-616. 